\title{
Neural estimation of kinetic rate constants from dynamic PET-scans
}

Fog, Torben L.; Nielsen, Lars Hupfeldt; Hansen, Lars Kai; Holm, Søren; Law, lan; Svarer, Claus; Paulson, Olaf

Published in:

Proceedings of the 4th IEEE Workshop Neural Networks for Signal Processing

Link to article, DOI:

10.1109/NNSP.1994.366004

Publication date:

1994

Document Version

Publisher's PDF, also known as Version of record

Link back to DTU Orbit

Citation $(A P A)$ :

Fog, T. L., Nielsen, L. H., Hansen, L. K., Holm, S., Law, I., Svarer, C., \& Paulson, O. (1994). Neural estimation of kinetic rate constants from dynamic PET-scans. In Proceedings of the 4th IEEE Workshop Neural Networks for Signal Processing (pp. 616-623). IEEE. https://doi.org/10.1109/NNSP.1994.366004

\section{General rights}

Copyright and moral rights for the publications made accessible in the public portal are retained by the authors and/or other copyright owners and it is a condition of accessing publications that users recognise and abide by the legal requirements associated with these rights.

- Users may download and print one copy of any publication from the public portal for the purpose of private study or research.

- You may not further distribute the material or use it for any profit-making activity or commercial gain

- You may freely distribute the URL identifying the publication in the public portal 


\title{
NEURAL ESTIMATION OF KINETIC RATE CONSTANTS FROM DYNAMIC PET-SCANS
}

\author{
Torben Fog, Lars Hupfeldt Nielsen and Lars Kai Hansen \\ CONNECT, Electronics Institute B349 \\ Technical University of Denmark, \\ DK-2800 Lyngby, Denmark \\ email: lkhansen@eileen.ei.dth.dk \\ Søren Holm, Ian Law, Claus Svarer, and Olaf Paulson \\ Dept. of Neurology, \\ The University Hospital of Copenhagen \\ DK-2100 Copenhagen $\emptyset$, Denmark
}

\begin{abstract}
A feed forward neural net is trained to invert a simple three compartment model describing the tracer kinetics involved in the metabolism of $\left[{ }^{18} \mathrm{~F}\right]$ flourodeoxyglucose in the human brain. The network can estimate rate constants from Positron Emission Tomography sequences and is about 50 times faster than direct fitting of rate constants using the parametrized transients of the compartment model.
\end{abstract}

\section{INTRODUCTION}

Positron Emission Tomography (PET) is an important tool for mapping of brain metabolism and functionality [2]. The primary target of PET is reconstruction of concentrations of certain radioactive tracers. The usefull tracers emit positrons that are locally annihilated to produce two $511 \mathrm{keV}$ gamma rays propagating in opposite directions. The 3D distribution of the tracer can be reconstructed from the geometric constraints of coincident counts, using standard techniques (filtered backprojection). An important class of tracers are chemically equivalent to compounds that enter the basic brain metabolism. By reconstructing such tracer distributions important aspects of brain metabolism have been revealed. Furthermore, by investigating the transient response to tracer injection, it is possible to identify fundamental kinetic rate constants. In this study we investigate the latter approach. The basic kinetic model was proposed by Sokoloff et al. [1]; in subsequent studies the model was used to estimate rate constant in lumped regions. In the 
work of Kanno et al. [3] pixel by pixel estimation of the rate-constants was introduced. This scheme has, however, not found wide spread use due to the complexity of the task of fitting the kinetic model transient to the large number of individual pixel transients. In this work we show how a neural network may substitute for such tedious parameter fitting procedures. The neural network system is trained to produce a smooth map relating a given transient with its most likely rate constants. This will provide a much faster estimation time for the individual pixel rate constants

\section{SOKOLOFF's KINETIC MODEL}

We consider here the kinetics of the compound $\left[{ }^{18} \mathrm{~F}\right]$ flourodeoxyglucose (FDG). The kinetics of this tracer is similar to glucose in the initial metabolic steps. It passes through the blood-brain barrier (BBB), and is phosphorylized in a process past the BBB analogous to glucose. Then it ceases to react further and is effectively trapped. The kinetics can be modelled by a compartemental model involving one compartment representing the tracer density in the arterial blood outside the $\mathbf{B B B}, C_{\mathrm{P}}^{*}$; one compartment representing the socalled precursor pool, $C_{\mathrm{E}}^{*}$; and finally a compartment representing the phosphorylized fraction behind the BBB, $C_{\mathrm{M}}^{*}$; see figure 1 . In current experiments the arterial concentrations are measured continuously along with the scan, hence the concentration $C_{\mathrm{P}}^{*}$ can be considered a control parameter for the compartment model.

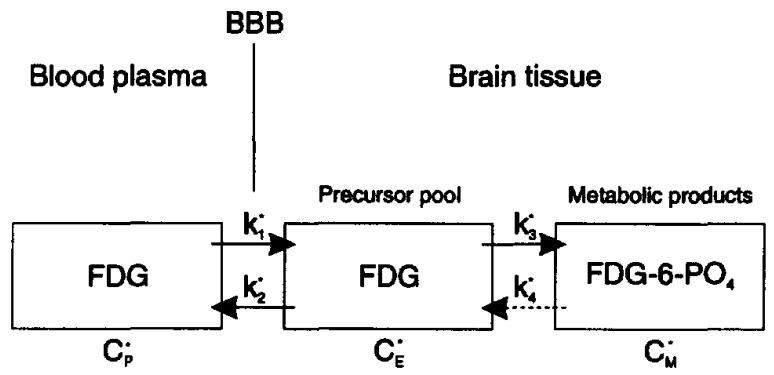

Figure 1: Sokoloff's three compartment model applied to phosphorylization of $\left[{ }^{18} \mathrm{~F}\right]$ flourodeoxyglucose (FDG). The star on the concentrations signifies that we consider tracer amounts and constants

Following the injection of the tracer, hence, the rise of the arterial blood concentration $C_{\mathbf{P}}^{*}$, the flow through the BBB starts. The measured PET tracer activity is the sum of the activities of the two compartments to the right of the BBB c.f. figure 1, 


$$
C_{i}^{*}=C_{E}^{*}+C_{M}^{*}
$$

The dynamics of the three compartment model is given by:

$$
\frac{d C_{i}^{*}}{d t}=\frac{d C_{E}^{*}}{d t}+\frac{d C_{M}^{*}}{d t}
$$

with

$$
\frac{d C_{M}^{*}}{d t}=k_{3}^{*} C_{E}^{*}
$$

and

$$
\frac{d C_{E}^{*}}{d t}=k_{1}^{*} C_{P}^{*}-k_{2}^{*} C_{E}^{*}-k_{3}^{*} C_{E}^{*}
$$

The reverse reaction rate constant $\left(k_{4}^{*}\right)$ corresponding to $k_{3}^{*}$ is neglected. These equations are straightforward to integrate providing the two time dependent concentrations,

$$
\begin{gathered}
C_{E}^{*}(t)=k_{1}^{*} e^{-\left(k_{2}^{*}+k_{3}^{*}\right) t} \int_{0}^{t} e^{\left(k_{2}^{*}+k_{3}^{*}\right) t^{\prime}} C_{P}^{*}\left(t^{\prime}\right) d t^{\prime} \\
C_{M}^{*}(t)=k_{1}^{*} k_{3}^{*} \int_{0}^{t}\left[e^{-\left(k_{2}^{*}+k_{3}^{*}\right) t^{\prime}} \int_{0}^{t} e^{\left(k_{2}^{*}+k_{3}^{*}\right) t^{\prime \prime}} C_{P}^{*}\left(t^{\prime \prime}\right) d t^{\prime \prime}\right] d t^{\prime}
\end{gathered}
$$

Following injection these solutions describe the transient activity in terms of the measured $C_{\mathrm{P}}^{*}(t)$ and the three rate constants. Conversely, for a given transient $C_{\mathrm{P}}^{*}(t)$ and for given measured sum of concentrations $C_{\mathrm{i}}^{*}(t)$ we may fit the three rate constants. An example is shown in figure 2. We use a simple least squares cost function for the fit, hence implicitly assuming Gaussian residuals. Optimization over the three parameters was carried out using a second order Newton scheme ${ }^{1}$

There are two different approaches from here. Up til now most studies assume that the rate constant are homogeneous in regions, see e.g. [1,2], and the rate constants are fitted from the regional average activity transient. Alternatively we can fit individual rate constants for each pixel in the reconstructed volume [3], and analyse for homogeneity. However, since it is quite tedious to fit the kinetic model, the latter approach has not found widespread use. In the upper panel of figure 3 we show the result of such a pixel by pixel fit. The artifacts outside the elliptic area of the brain are due to the reconstruction scheme used (Filtered backprojection).

The database used for these experiments are PET data collected at the PET center at Rigshospitalet, Copenhagen. The subject described in this paper is a 43 year old woman with multiple sclerosis. Data are aquired on a

${ }^{1}$ Based on the solution to the kinetic model it is straightforward to compute the second derivatives. 
GE4096 plus (General Electric Medical Systems), sampling 15 slices simultaneously. The dynamic scans after injection of $200 \mathrm{MBq}$ F-18 labelled FDG are performed over 60 minutes, yelding 34 contiguous time frames of increasing duration in order to provide a reasonable sampling of the $C_{i}^{*}$ curve: $(10 @ 6$ sec; $3 @ 20 \mathrm{sec} ; 8 @ 60 \mathrm{sec} ; 5 @ 120 \mathrm{sec} ; 8 @ 300 \mathrm{sec}$ ). A single such curve is shown in figure 2 . Images were reconstructed in $128 \times 128$ matrices $\left(2 \mathrm{~mm}^{2}\right.$ pixels) by standard Filtered backprojection (Ramp filter with Hann window). Correction for attenuation is based on a separate transmission scan with a rotating Germanium pin source. For further introduction to PET scan techniques see e.g., [2]

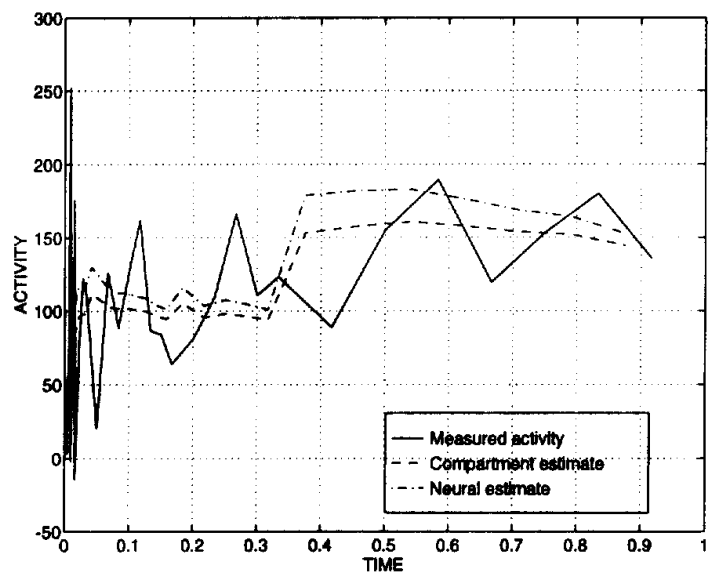

Figure 2: $C_{i}^{*}(\mathrm{t})$ as measured by PET for a single pixel, as produced by the kinetic compartment model with the fitted rate constants, and as estimated by the neural network.

To avoid the tedious fitting procedure we here investigate the possibility of identifying the inverse model of the kinetics: we search for a map that provides an estimate of the three rate constants for a given observed transient. Our basic vehicle will be a simple feed-forward network.

What should be used for inputs?. The PET transients depend, c.f. equations (5-6), on the rate constants and on the time dependent arterial concentration $\left(C_{\mathrm{P}}^{*}\right)$. If we want to generalize from one set of pictures to another set (possibly another subject) we would need to provide both the observed PET transient and $C_{\mathrm{P}}^{*}(t)$. This will be pursued in future studies. In this work we tentatively train the network to predict the rate constants for pictures of a single sequence of PET images, hence, $C_{\mathrm{P}}^{*}$ is the same for all pixels and we need not provide it as input. In particular we train the network on transients from a small subsample of one slice of the PET volume scan Subsequently we apply the trained network to get the rate constants for the (large) remaining set of pixels. 

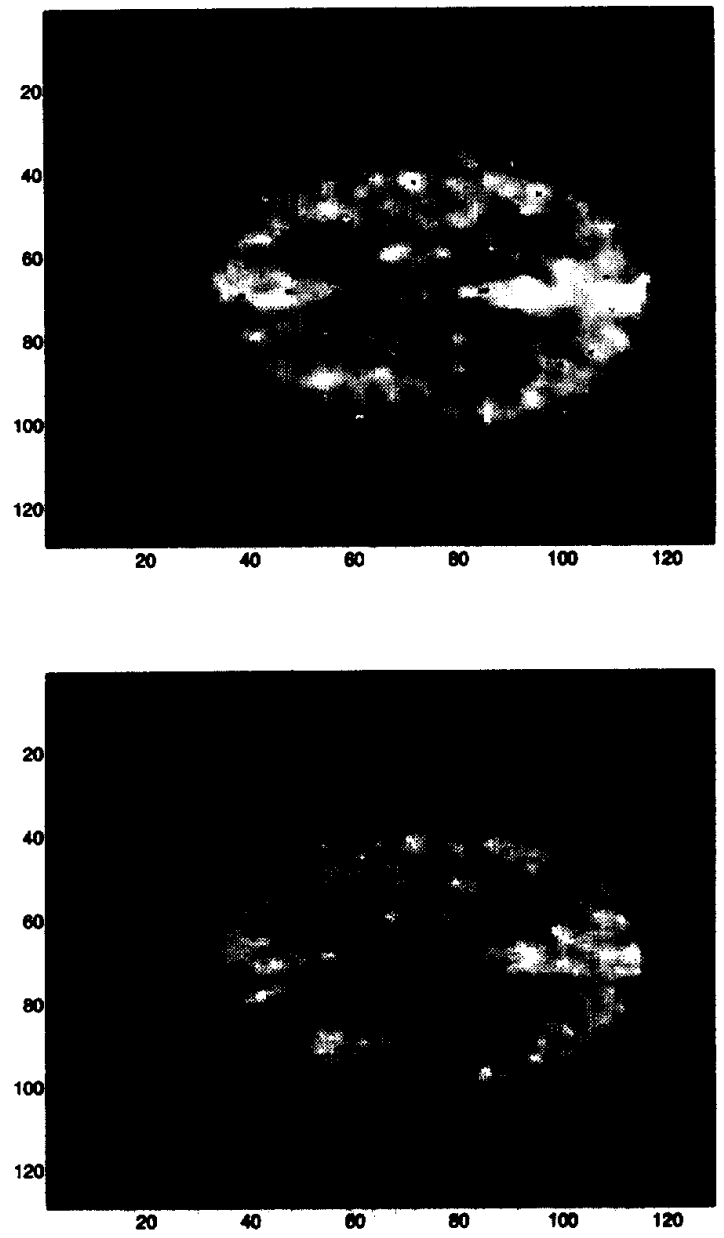

Figure 3: Image (slices) showing the $k_{1}^{*}$ rate constant as determined by fitting the kinetic model (pixel by pixel) using a second Newton scheme (upper panel) and as determined by the neural net operating as inverse model for the kinetics (lower panel). The artifacts outside the elliptic area of the brain are due to the "Filtered backprojection" algorithm used for reconstruction. 


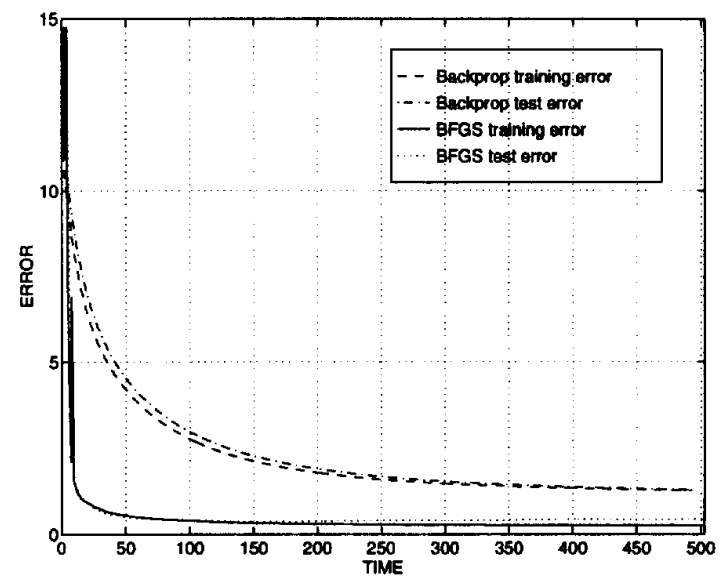

Figure 4: Comparison of time developments for the training process of standard Backpropagation and the BFGS scheme.

\section{NETWORK DESIGN}

The network considered is a standard feed forward net with a single layer of hidden units. The activation function of the hidden units are hyperbolic tangents, while the output units are linearly activated. The particular network for this study had 34 inputs corresponding to the activity transient of a given pixel. The net had ten hidden units and three output units. We trained the network by a pseudo second order scheme, the Broyden-Fletcher-GoldfarbShanno (BFGS) algorithm see e.g. [4]. This algorithm is a variable metric method that constructs a sequence of matrices approximating the inverse Hessian. Using BFGS instead of e.g. standard Backpropagation [5] provides a significant speed-up. This is quantified in figure 4 , showing the time development of the training process with backprop and with the BFGS method respectively. Note the scale is in arbitrary CPU time units not iterations, since a backprop iteration is faster than a BFGS iteration.

For most adaptive systems the objective is not to learn the training set, but rather to perform well on a much larger set of conceivable inputs, i.e., generalization. The generalization ability depends on architecture and on size of the training set. Hence, for a given architecture, it is important to estimate the necessary number of training cases (pixels) to obtain good generalization. This relation is quantified by the so-called learning curve of the architecture as shown in figure 5 . We note that a mere 4000 pixels are needed to obtain the asymptotic level of the test error.

Finally we apply the trained network to produce a full estimate of a "slice" of the PET scan as presented in the lower panel of figure 3. It is quite remarkable that the image quality of the rate constants reconstructed by the 


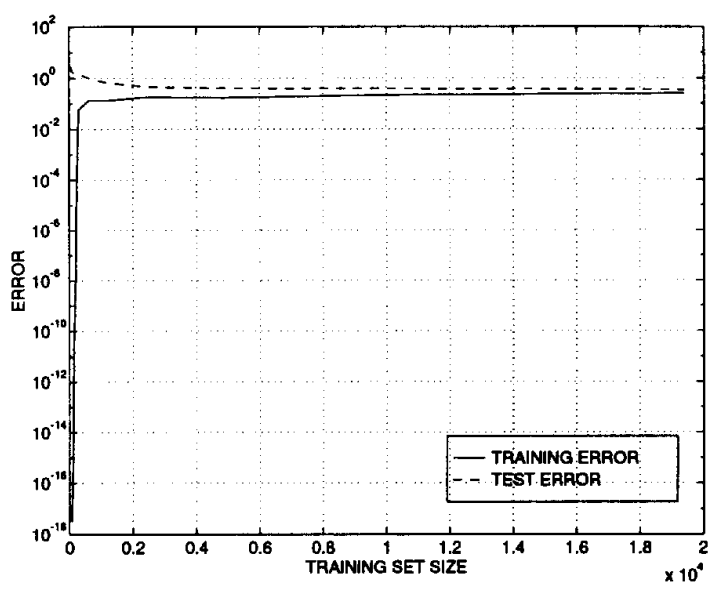

Figure 5: Learning curve for the feed forward net trained by BFGS, approximately 5000 pixels are needed to reach the asymptotic generalization level (about a quarter of the pixels in an image (slice)).

networks inverse model is less noisy than quality obtained from the fitting procedure. The reason is that the network capacity is limited by the inherent constraints of the network architecture, while the Newton fit can lead to arbitrarily (wrong) rate constants for a given pixel if the transient is very noisy. We also note that the execution time for the feed forward network is about two percent of the average time needed to obtain the kinetic constants by fitting the transients with the Newton method.

\section{CONCLUSION}

We have shown how a feed forward net may be used for identification of the inverse model for three compartment PET tracer kinetics. Not only is the use of the feed forward net significantly faster than fitting the kinetic model, but our tentative results seem to indicate that the rate constant distribution is regularized by the constraints imposed by the network architecture.

\section{ACKNOWLEDGMENTS}

This research was supported by the Danish Natural Science and Technical Research Councils through the Computational Neural Network Center (CONNECT). 


\section{REFERENCES}

[1] L. Sokoloff, M. Reivich, C. Kennedy, M.H. Des Rosiers, C.S. Patlak, K.D. Pettigrew, O. Sakurada, and M. Shinohara: "The C-14-Deoxyglucose Method for the Measurement of local Cerebral Glucose Utilization: Theory, Procedures and Normal Values in the Conscious and Anesthetized Albino Rat" Journal of Neurochemistry 28 897-916, (1977).

[2] M.E. Phelps: "Positron Emission Tomography (PET)". In: J.C. Maziotta and S. Gilman (Eds.): Clinical Brain Imaging, Principles and Applications, F.A. Davis Company, Philadelphia (1992).

[3] H. Sasaki, I. Kanno, M. Murakami, F. Shishido, and K. Uemuda: "Tomographic Mapping of KInetic Rate Constants in the FDG model Using Dynamic PET". Journal of Cerebral Blood Flow 6 447-454 (1986).

[4] W. Press et al.: Numerical Recipes in ' $C$ '. Cambridge University Press.

[5] D. E. Rumelhart and J. L. McClelland: "Back-propagation of errors" in Parallel Distributed Processing. Explorations in the Microstructure of Cognition, Vols. 1-2, MIT Press, Cambridge, Massachusetts (1986). 